\title{
Effective robotic assistive pattern of treadmill training for spinal cord injury in a rat model
}

\author{
BO-LUN ZHAO ${ }^{1}$, WEN-TAO LI $^{2}$, XIAO-HUA ZHOU ${ }^{2}$, SU-QIAN WU ${ }^{2}$, \\ HONG-SHI CAO ${ }^{1}$, ZHU-REN BAO ${ }^{2}$ and LI-BIN AN ${ }^{1}$ \\ ${ }^{1}$ Department of Clinical Nursing, School of Nursing, Jilin University, Changchun, Jilin 130021; \\ ${ }^{2}$ Department of Clinical Nursing, School of Nursing, Dalian University, Dalian, Liaoning 116622, P.R. China
}

Received September 28, 2017; Accepted January 24, 2018

DOI: $10.3892 /$ etm.2018.5822

\begin{abstract}
The purpose of the present study was to establish an effective robotic assistive stepping pattern of body-weight-supported treadmill training based on a rat spinal cord injury (SCI) model and assess the effect by comparing this with another frequently used assistive stepping pattern. The recorded stepping patterns of both hind limbs of trained intact rats were edited to establish a 30-sec playback normal rat stepping pattern (NRSP). Step features (step length, step height, step number and swing duration), BBB scores, latencies, and amplitudes of the transcranial electrical motor-evoked potentials (tceMEPs) and neurofilament 200 (NF200) expression in the spinal cord lesion area during and after 3 weeks of body-weight-supported treadmill training (BWSTT) were compared in rats with spinal contusion receiving NRSP assistance (NRSPA) and those that received manual assistance (MA). Hind limb stepping performance among rats receiving NRSPA during BWSTT was greater than that among rats receiving MA in terms of longer step length, taller step height, and longer swing duration. Furthermore a higher BBB score was also indicated. The rats in the NRSPA group achieved superior results in the tceMEPs assessment and greater NF200 expression in the spinal cord lesion area compared with the rats in the MA group. These findings suggest NRSPA was an effective assistive pattern of treadmill training compared with MA based on the rat SCI model and
\end{abstract}

Correspondence to: Professor Li-Bin An, Department of Clinical Nursing, School of Nursing, Jilin University, 965 Xinjiang Street, Changchun, Jilin 130021, P.R. China

E-mail: anlibin2001@163.com

Abbreviations: SCI, spinal cord injury; NRSPA, normal rat stepping pattern assistance; MA, manual assistance; tceMEPs, transcranial electrical motor-evoked potentials; NF200, neurofilament 200; BWSTT, body weight-supported treadmill training; RRMPS, rodent robotic motor performance system; MD, mean density; AF, area fraction

Key words: rat model, robotic assistive pattern, spinal cord injury, treadmill training this approach could be used as a new platform for animal experiments for better understanding the mechanisms of SCI rehabilitation.

\section{Introduction}

Activity-based rehabilitation is a promising strategy for improving functional recovery after spinal cord injury (SCI) by enhancing the growth of descending and ascending fiber tracts around or through the lesion, restoring the levels of neurotrophins $(1,2)$, and preventing secondary damage following spinal cord injury (3). For decades, activity-based rehabilitation strategies aimed at improving locomotor functions have been extensively investigated in humans and in animal models with partial or complete SCI. Parameters, such as type of training (4-6), amount of training (7) and speed (8), training program duration $(9,10)$, and combinations of rehabilitation methods (11-13) have been assessed to evaluate the outcome of different activity-based rehabilitation strategies, which have identified the potential of specific training paradigms to enhance locomotor function after SCI.

Walking recovery is the goal of patients who experience SCI (14). Marked restoration of stepping in the paralyzed hindlimbs in spinalized animals has been reported following treadmill training (15-17). Therefore, retraining for walking after SCI has largely focused on mass practice with repetitive stepping on a treadmill or over ground (18). Body-weight-supported treadmill training (BWSTT) is a type of step training therapy that incorporates treadmill training with body weight support. BWSTT has demonstrated improvements in walking in animal models with SCI (19), particularly in rats. Due to the positive outcomes $(100 \%$ in rats and cats for treadmill training and $75 \%$ for BWSTT in rats and $100 \%$ in cats) (19), especially restoration of walking, treadmill training, and BWSTT have been frequently applied in animal models with SCI.

Treadmill training and BWSTT are generally introduced in the early phase after SCI, which suggests that the timing of training may be important, since most neurological recovery occurs within the first month after injury. A long delay (20) between injury and the beginning of training appears to reduce the beneficial effects of training regimens on the regrowth of nerve fibers and the plasticity of the spinal cord $(19,20)$. Furthermore, an early initiation of the 
exercise plan can attenuate muscle atrophy and bone loss (21). However, the primary limitation of step treadmill training is that it requires a certain residual function, which is typically absent during the early phase of SCI. Therefore, assistance, either by a robotic device or manually, is initially required. Studies have shown that robotic devices can facilitate training by providing precise control over limb weight bearing and improve locomotor functions (22) and cardiovascular fitness during exercises (23). However, to date, few animal or human studies have investigated the effects of assistive training stepping patterns. Previous studies using robotic arms as assistive tools did not report the details of the assistive training patterns or methods (17). Several studies used manually recorded assistive training patterns $(16,24)$. Lee et al first reported the use of manual assistance (MA) for training patterns by electric robotic arms, to provide assistance to the hindlimbs of spinally contused rats during BWSTT, and demonstrated that assist-as-need (AAN) step training resulted in better hindlimbs stepping performance than full assist (FA) step training (24). However, the primary deficit of MA is that it is considerably different from the normal gait pattern of the animals and these differences may limit the generation of independent stepping or coordination of the limbs. In theory, the aim of gait training is to assist patients to recover normal step function; therefore, it should typically mimic the normal step pattern. To overcome this limitation, a novel algorithm has been implemented, which can synchronize the robotic device with the actual motion of the individual in real-time (25) or trajectory tracking (26), establishing subject-specific trajectories (26). Recently, a new training pattern has been developed for hemiparetic patients that records the movement of the unimpaired contralateral limb to train the impaired limb (27). However, the effectiveness of robotic step training relative to manually assisted step training is unclear (28). It is important to compare these two assistance training patterns in animal models as the first step to identify the best training pattern to enhance the rehabilitation effect of BWSTT following SCI.

Early initiation of step training and effective assistance are critical factors that influence the outcomes of BWSTT after SCI. Robotic devices have the advantage of providing repetitive, systematic, prolonged gait training sessions (29) and reducing the labor of therapists. Therefore, the establishment of an effective robotic assistance pattern of treadmill training is vital for early initiation of training. The purpose of this study was to examine and identify the effectiveness of different assistance patterns for spinal cord injury in a rat model.

\section{Materials and methods}

Animals. Procedures involving animals and their care were conducted in accordance with the institutional guidelines of the local Ethics Committee for Animal Research at Dalian University, and complied with the National Institutes of Health Guidelines for the Care and Use of Laboratory Animals. Forty

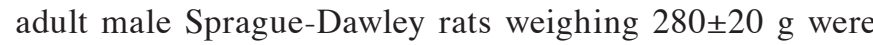
obtained from the Experimental Animal Center of Dalian Medical University (Dalian, China). The rats were raised in separated cages under controlled temperature $\left(22-24^{\circ} \mathrm{C}\right)$ and humidity (40-50\%) with a standardized 12-h light-and-dark cycle and free access to food and tap water.
Eight of the 40 rats were used for normal step pattern recording and electrophysiological baseline testing. The remaining 32 rats were randomly assigned to the following 4 groups (8 rats/group): The sham group (including rats who underwent laminectomy with no injury to the spinal cord and without BWSTT), the sedentary group (SCI but without treadmill training), the normal rat stepping pattern assistance (NRSPA) group (BWSTT for 3 weeks beginning at 3 days after SCI with NRSPA), and the MA group (BWSTT for 3 weeks beginning 3 days after SCI with MA).

The study has been approved by the Ethics Committee of Affiliated Zhongshan Hospital of Dalian University (2017082).

Surgery and spinal cord contusion injury. Rats were anesthetized with $7 \%$ chloral hydrate $(350 \mathrm{mg} / \mathrm{kg})$ intraperitoneally. The rats were placed in the supine position on a surgical table, and the spinous process of the T10 thoracic vertebra was located by palpating the ribs. The dorsal skin was shaved and disinfected with iodine at the incision site. A $20-\mathrm{mm}$ midline incision was made in middle of the thoracic region of the dorsum with a fine scalpel and the overlying fascia and muscles were retracted to expose the dorsal surface of the T10 vertebrae. The spinous process and the dorsal parts of vertebral plate of T10 were resected with bone rongeurs until the dorsal epidural surface of the spinal cord was exposed. Twenty-four of the 32 rats received a severe mid-thoracic contusion of the spinal cord, while in the remaining 8 rats, the T10 lamina was opened, exposing the spinal cord, without causing spinal cord contusion (the sham group). Spinal cord contusion injury was made by dropping a $10-\mathrm{g}$ rod from a distance of $25 \mathrm{~mm}$ to directly impact the dorsal surface of the exposed spinal cord. The rod was removed immediately following the injury. Indicators of successful contusion include the red and swollen appearance of the local spinal cord, fluttering of both hindlimbs immediately after the impact, and bilateral hindlimbs paralysis when the rats regained consciousness. The surrounding tissues were then closed layer by layer with surgical sutures. After the surgery, the rats were immediately placed on warm water pads until they regained consciousness. Penicillin $(160 \mathrm{mg} / \mathrm{kg})$ was administered intraperitoneally at the end of the surgery for 3 consecutive days to prevent infection. Manual bladder expression was performed twice daily until the rats recovered spontaneous micturition.

Training procedures after SCI. Establishment of stepping play-back pattern for assistance during training. The Rodent Robotic Motor Performance System (RRMPS) (Robomedica, Inc., Mission Viejo, CA, USA) was used to train and test treadmill stepping in the rats. RRMPS is the updated version of Rodent Robot 3000 (Robomedica, Inc.) (24). RRMPS has a motorized-variable-speed treadmill, two robotic arms, and a body-weight-support arm. The rats are secured to the weight-supporting arm of the robotic device with a vest. The ankles of the rats were attached to the distal ends of the robotic arms with rubber loops that wrapped around the ankle. RRMPS can record stepping patterns and playback the recording stepping patterns.

First, the stepping patterns of the intact rats were recorded. Eight rats [Basso, Beattie, and Bresnahan (BBB) score=21]were trained to adapt to RRMPS for 4 walking sessions per day, lasting 
approximately $15 \mathrm{~min} / \mathrm{session}$, separated by a 15 -min resting period in the, with food rewards after each training session. After 1 month of training, the rats could successfully step on the treadmill. Repetitive stepping recordings were performed using RRMPS at a treadmill speed of $7 \mathrm{~cm} \cdot \mathrm{sec}^{-1}$ and $80 \%$ weight support with both forelimbs and hindlimbs touching the surface of the treadmill at the same time to maintain the normal quadrupedal locomotion pattern of the rats. The rats were observed and recorded until the most stable stepping pattern was visualized on the computer. The recorded stepping patterns were analyzed and edited using the analysis software provided by RRMPS. A 30-sec consecutive hindlimbs step playback-pattern of a normal rat was determined (Fig. 1).

To test the reliability and constancy of the recorded step pattern, 6 primary parameters related to step characters were analyzed: Step length (horizontal movements of the toe between two successive paw contacts of the same limb); step height (vertical movements of the toe between two successive paw contacts of the same limb); step cycle duration (the time between two successive contact points of the same paw on the treadmill); stance duration (the time when the foot is on the treadmill during 1 step, i.e., from the end of 1 step to the beginning of the next step of the same foot); swing duration (the time during which the foot is lifted during 1 step); and stance duration/swing duration (the ratio of stance time and swing time in $1 \mathrm{step})$. The t-test was used to determine significant differences between right and left limbs in this step pattern based on the six parameters, and no significant differences ( $P>0.05)$ were found. The coefficients of variance $(\mathrm{CV})$ of the six parameters of the same limb were all under $15 \%$, indicating the consistency and stability of the step pattern (Table I).

Training protocol. Because the injury did not affect the forelimbs of the SCI rats, forelimb stepping following the speed of the treadmill can contribute to body support and interappendicular coordination. Quadrupedal stepping is the normal stepping pattern rather than bipedal stepping (30). Hence the quadrupedal stepping protocol was adopted in this study.

One week prior to surgery, all the rats were familiarized with RRMPS (including the vest, the robotic arms, the body support system and the speed of the treadmill). The rats in the NRSPA and MA groups started BWSTT 3 days after the surgery. BWSTT of the SCI rats was performed for $15 \mathrm{~min}$ twice per day with a 15 -min break between sessions. The total duration of training was 3 weeks with 5 days of training per week. The speed of the treadmill was maintained constant $\left(7 \mathrm{~cm} \cdot \mathrm{sec}^{-1}\right)$ and $80 \%$ of the body weight was supported. The recorded normal rat stepping pattern (NRSP) performed in a repetitive loop was implemented on the hindlimbs of the rats from the NRSPA group by the robotic arms controlled by the RRMPS software (version 1.0; Robomedica Inc.). MA by an experienced trainer was implemented as required to the hindlimbs of the rats from MA group.

Locomotor function assessment and analysis step detection. Locomotor function assessment and analysis step detection was performed for all the groups before BWSTT (3 days post-surgery) and at the termination of BWSTT. BBB scores were assessed 4 times for all groups: 3 days post-surgery and at the end of every week BWSTT.
Table I. Descriptive statistical data of 30-sec normal rat step mode recorded by the rodent robotic motor performance system (mean $\pm \mathrm{SD})$.

\begin{tabular}{lccc}
\hline Data classification & & $\begin{array}{c}\text { Left } \\
\text { hindlimb }\end{array}$ & $\begin{array}{c}\text { Right } \\
\text { hindlimb }\end{array}$ \\
\hline Step length (mm) & mean \pm SD & $63.51 \pm 4.79$ & $63.80 \pm 7.13$ \\
& CV\% & 7.54 & 11.26 \\
Step height (mm) & mean \pm SD & $23.36 \pm 3.17$ & $23.17 \pm 3.03$ \\
& CV\% & 13.58 & 13.09 \\
One step cycle (sec) & mean \pm SD & $1.45 \pm 0.23$ & $1.46 \pm 0.22$ \\
& CV\% & 12.33 & 12.89 \\
Stance duration & mean \pm SD & $1.02 \pm 0.21$ & $1.01 \pm 0.19$ \\
of one step (sec) & CV\% & 12.89 & 12.20 \\
Swing duration & mean \pm SD & $0.43 \pm 0.03$ & $0.44 \pm 0.02$ \\
of one step (sec) & CV\% & 14.30 & 13.64 \\
Stance/Swing & mean \pm SD & $2.37 \pm 1.04$ & $2.23 \pm 1.01$ \\
in one step cycle & CV\% & 14.10 & 14.54 \\
\hline
\end{tabular}

$\mathrm{CV}$, coefficient of variation; SD, standard deviation.

Step detection and parameters analysis by RRMPS. No robotic assistance was provided to the ankles during the tests. The stepping patterns of the rats were consecutively recorded for $30 \mathrm{sec}$ with a treadmill speed of $7 \mathrm{~cm} \cdot \mathrm{sec}^{-1}$ and $80 \%$ weight support. The robotic arms of RRMPS recorded the kinematic features of stepping, including step length and height, swing duration, and successful step numbers, which can quantitatively reflect the subtle locomotor function recovery of the SCI rats.

A successful step cycle was identified by RRMPS analysis software (Robomedica, Inc.), which was defined as a minimum $10 \mathrm{~mm}$ in step length and $3 \mathrm{~mm}$ in step height (24). The data for each stepping kinematic feature of the rats were averaged and stored on a computer for further analysis.

$B B B$ score. The BBB score consists of an ordinal scale from 0 (flaccid paralysis) to 21 points (normal gait) (31). Higher scores are associated with better locomotor function. The BBB score was evaluated by two experienced investigators who were double blinded. During the evaluation, the rats were allowed to move voluntarily in a square plastic box $(100 \times 100 \times 4 \mathrm{~cm})$ without cover for $4 \mathrm{~min}$, and the movements of hindlimb were observed; BBB scores were averaged for each group.

Neurophysiological assessment. Transcranial electrical motor-evoked potentials (tceMEPs) were obtained using the EMG/EP System NDI-094 (Haishen Medical Electronic Instrument Co., Ltd., Shanghai, China). Typical waveforms (Fig. 2) and parameters of tceMEPs were recorded in intact rats as the baseline. The tceMEPs for all experimental groups were recorded twice when the BWSTT training was initiated (3 days post-surgery) and terminated (3 weeks after the initiation of BWSTT).

For tceMEPs, a dosage of $7 \%$ chloral hydrate $(350 \mathrm{mg} / \mathrm{kg}$ ) was administered to the rats intraperitoneally to 

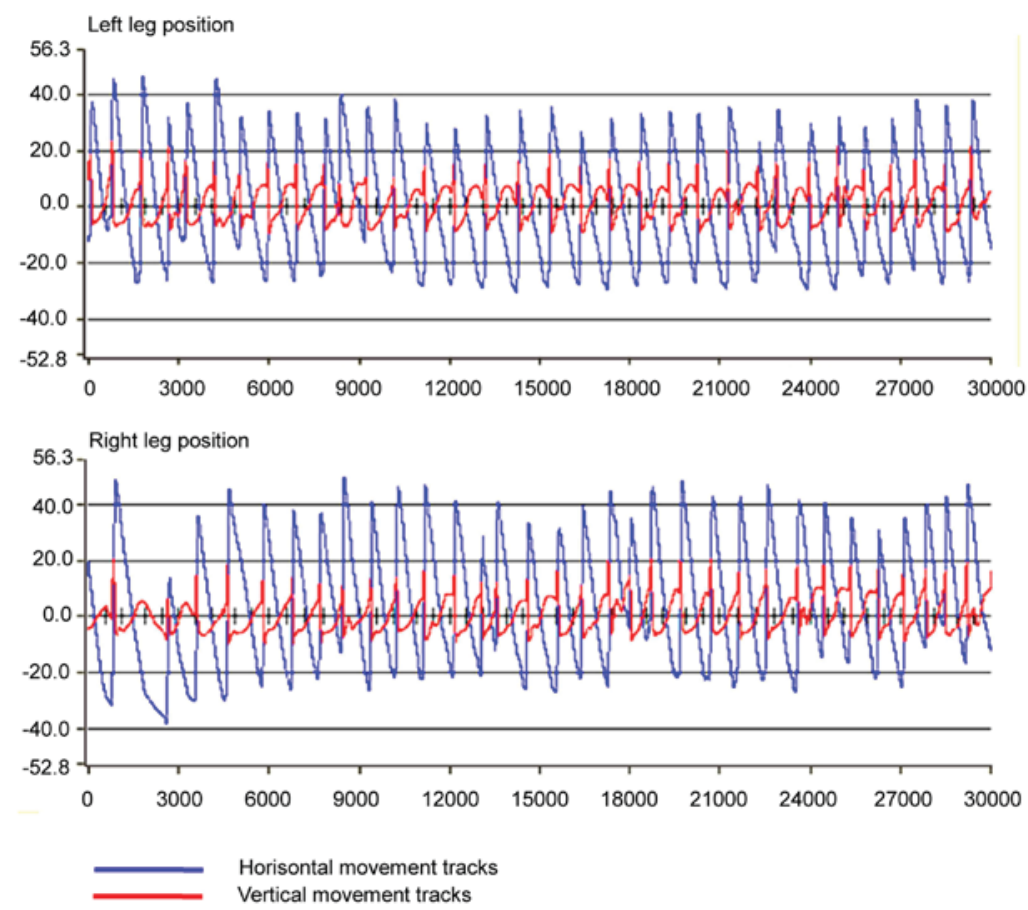

Figure 1. Image of paw positions of the right and left limb during the $30 \mathrm{sec}$ recording of the normal step pattern of intact rats (NRSP). The blue lines represent the horizontal movement trajectory of the paws of the hindlimbs and the red lines represent the vertical movement tracks of the paws of the hindlimbs. The horizontal ordinate shows the distance of the movements $(\mathrm{mm})$ and the vertical ordinate shows the time course (sec). Blue lines going upward indicate the foot moving forward and red lines going upward indicate the lifted foot. Conversely, lines going downward indicate the foot moving backwards and foot placed down. The distance from the top point to the bottom point of the blue and red lines in 1 step cycle represents the maximal step length and the maximal step height, respectively.

induce anesthesia. The head region of the rats was shaved and disinfected with $75 \%$ ethyl alcohol. The stimulating needle was positioned beneath the scalp, $2 \mathrm{~mm}$ anterior to the coronal suture and $2 \mathrm{~mm}$ lateral to the sagittal suture. The reference electrode was placed $0.5 \mathrm{~cm}$ posterior to the recording electrode. The ground electrode slice was positioned on the skin of left forelimbs. An intramuscular mono-polar recording needle was inserted into the belly of the gastrocnemius muscle in the right hindlimb. The reference electrode was inserted into the distal tendon (32).

Monopolar electrical stimulation was used to induce transcranial electrical stimulation, with a current intensity of 15-20 mA, a pulse width of $0.2 \mathrm{msec}$, frequency of $2 \mathrm{~Hz}$. A total of 50 superimposed traces were recorded, with a scanning speed of $3 \mathrm{msec} / \mathrm{D}$ and sensitivity of $1 \mathrm{mV} / \mathrm{D}$. Stimulation intensity was increased gradually, until the movement of both forelimbs developed and a clear MEPs pattern was detected. The latency and amplitude of tceMEPs and the tceMEPs waveforms were recorded and stored on the computer for analysis.

Tissue preparation and immunohistochemistry. Immediately following the last locomotor function and electrophysiological assessments, the rats were perfused transcardially with $4 \%$ paraformaldehyde in $0.1 \mathrm{~mol} / 1$ phosphate buffer saline (PBS, $\mathrm{pH} 7.4$ ) at $4^{\circ} \mathrm{C}$ for $20-30 \mathrm{~min}$ after deep anesthesia with $7 \%$ chloral hydrate $(350 \mathrm{mg} / \mathrm{kg})$. The spinal cords were harvested and post-fixed in the same fixation fluid for $48 \mathrm{~h}$ (33). The spinal cords were cut into $1.0-\mathrm{cm}$ long pieces along the rostrocaudal axis with the lesion area at the center. Paraffin specimens were prepared by a paraffin embedder $(\mathrm{EG} 1150 \mathrm{H})$

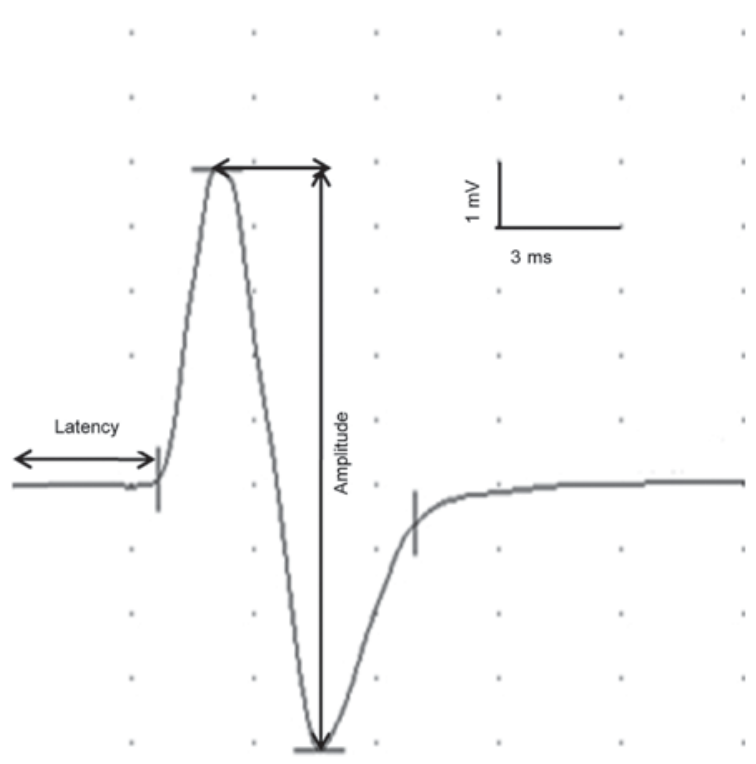

Figure 2. tceMEPs waveform of intact rats recorded in the gastrocnemius The latency (ms) was the interval from administration of the electrical stimulation to the starting point of the initiation of the tceMEPs waveform. The amplitude $(\mathrm{mV})$ was defined as the height from the positive peak to the negative peak of the waveform. tceMEPs, transcranial electrical motor-evoked potentials.

after dehydration using semi-enclosed bench top tissue processor (TP1020) and cooled on the cold plate (EG1150C; all from Leica Microsystems $\mathrm{GmbH}$, Wetzlar, Germany) at a constant temperature of $5^{\circ} \mathrm{C}$ for $30 \mathrm{~min}$. Twenty-five adjacent serial $6-\mu \mathrm{m}$ thick cross-sectional paraffin sections were 


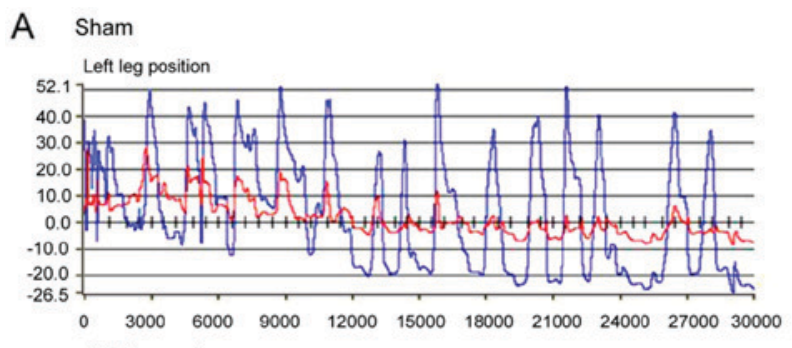

B Sedentary
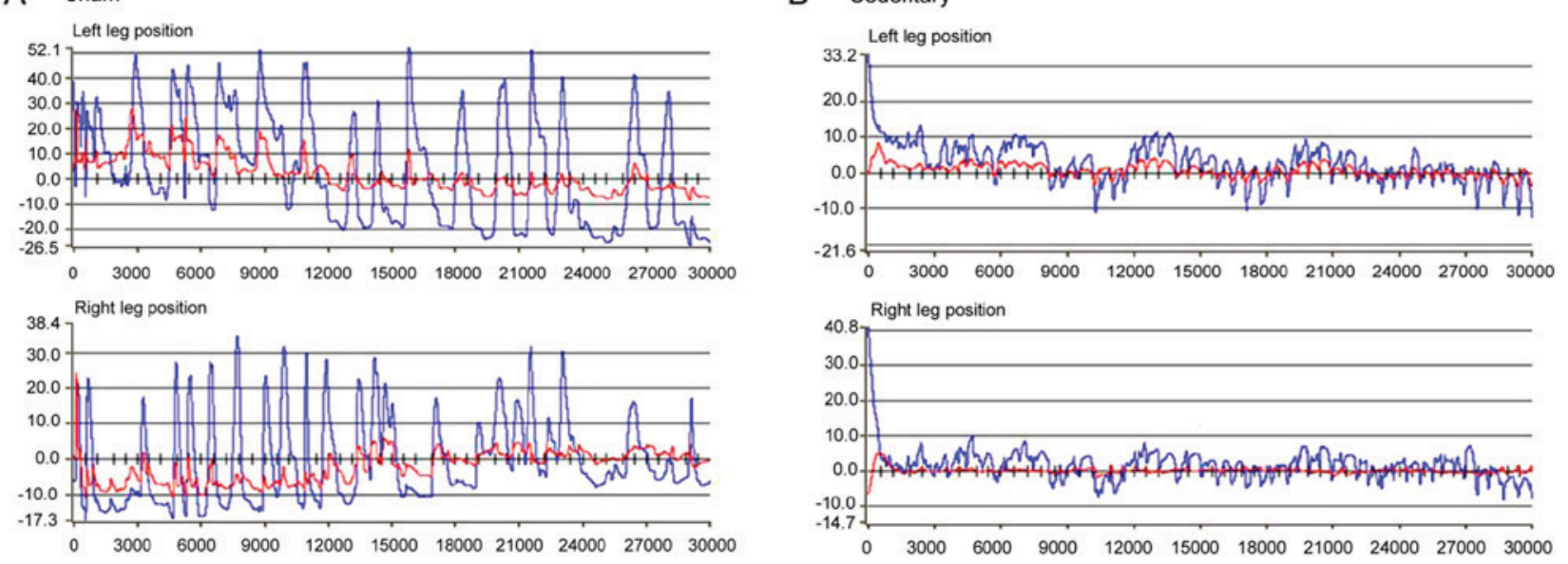

C NRSPA

D MA
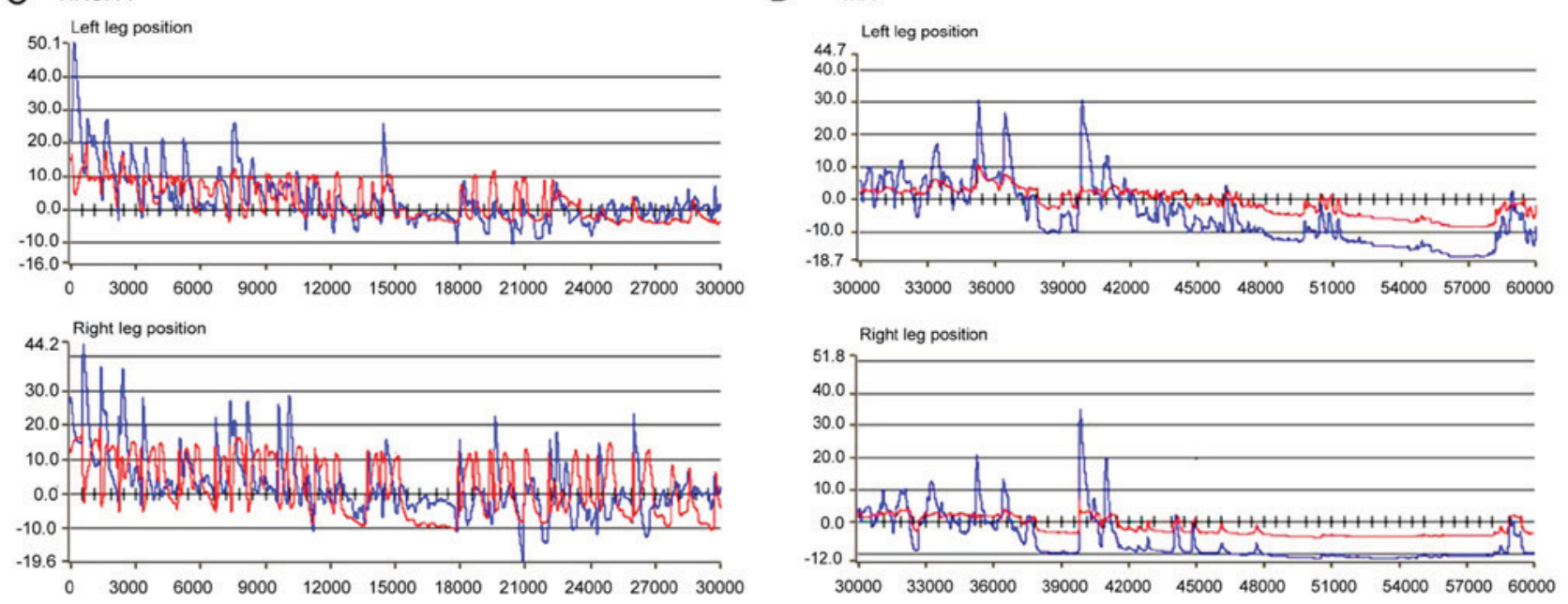

Horisontal movement tracks

Vertical movement tracks

Figure 3. Representative images of paw positions of the right and left limb during 30-sec testing for each experimental group after 3 weeks of BWSTT. (A-D) represent the trajectories of the right and left paws' movements for sham, sedentary, NRSPA and MA groups, respectively. For detailed explanations see Fig. 1. According to the amplitudes of the blue lines (horizontal movements) and red lines (vertical movements), the trajectories of the two BWSTT groups (NRSPA and MA) are obviously lower than that of the sham group and greater than that of the sedentary group. Furthermore, by comparison of C and D, the movements were greater in the NRSPA group than in the MA group, particularly in relation to the red lines (vertical movements). BWSTT, body-weight-supported treadmill training; NRSPA, normal rat stepping pattern assistance; MA, manual assistance.

obtained at the epicenter of the lesion area for each spinal cord sample using manual rotary microtome (RM2235; Leica), and every 5th section was selected for immunohistochemistry. The procedure for immunocytochemistry has been described previously (34) and in this study, we chose fluorescent staining instead of DAB. The primary antibody was neurofilament 200 (NF200) antibody (BM0100) diluted 1:200 and the secondary antibody was anti-GAPDH rabbit monoclonal antibody (M00227) (both from BosterBio, Pleasanton, CA, USA) diluted 1:1,000. Digital photomicrographs of 5 visual areas in the dorsal horn in a section were taken using a fluorescent microscope (Ci-1; Nikon, Tokyo, Japan) with a $20 \mathrm{X}$ objective lens under a constant exposure condition for all the sections. Image-Pro Plus 6.0 software (Media Cybernetics, Silver Springs, MD, USA) was used to analyze the area, integrated density (ID), and area fraction (AF) of NF200+ expression of each image after normalization of the background intensity. The mean density (MD) of NF200-labeled axons for each sample was calculated for further statistical analysis.

Statistical analysis. All data were analyzed using SPSS 19.0 (SPSS, Inc., Chicago, IL, USA). Mean and standard deviation (SD) were used to describe the sample parameters. Factorial design ANOVA was used to determine the source of variation in step analysis. Multigroup comparisons of the means were carried out by one-way analysis of variance (ANOVA) with post hoc contrasts by least significant difference (LSD). $\mathrm{P}<0.05$ was considered to indicate a statistically significant difference.

\section{Results}

Rats in the NRSPA group achieved better stepping quality than those in the MA group. Before the initiation of BWSTT, the hindlimbs of the sham rats could step, although the number 

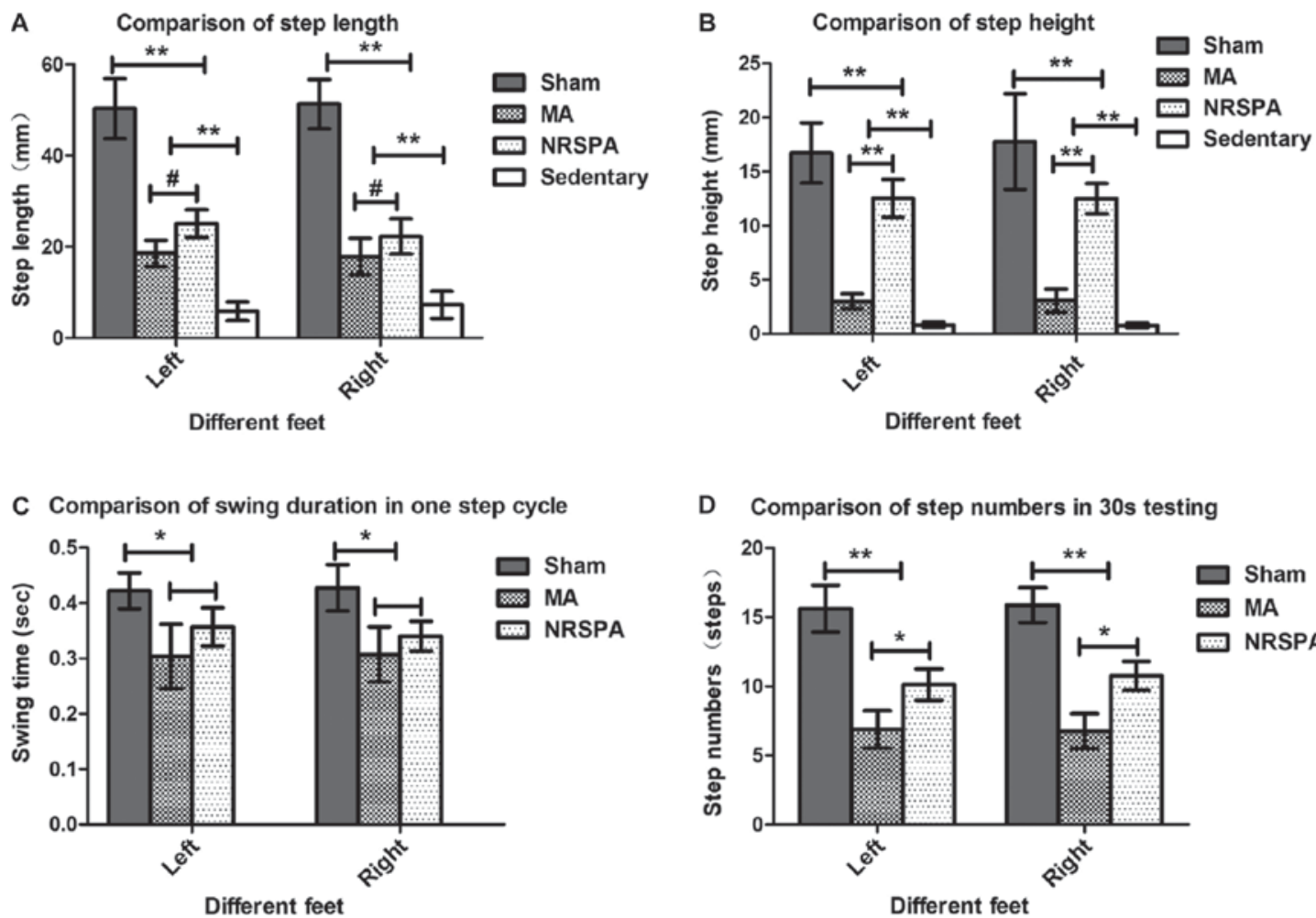

D Comparison of step numbers in 30 s testing

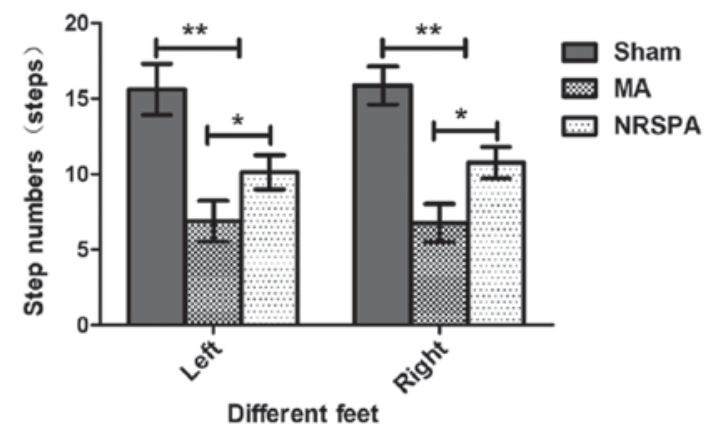

Figure 4. Comparisons of the step parameters analysis between different groups during 30-sec testing after 3 weeks of BWSTT. The charts represent the outcomes of step parameters statistical analysis by comparison between all the experimental groups for step length (A), step height (B), swing duration in 1 step cycle (C), and step numbers (D) in the 30-sec testing. The sedentary group was not included in the analysis of swing duration and step number as they did not meet the criterion for a step. One-way ANOVA and LSD were used for statistical analysis and $\mathrm{P}<0.05$ was statistically significant. ${ }^{*} \mathrm{P}<0.05$, ${ }^{*} \mathrm{P}<0.01$, ${ }^{* *} \mathrm{P}<0.001$. From the above parts, the sham group had the best outcomes for all the parameters of step analysis compared to the NRSPA, MA and sedentary groups $(\mathrm{P}<0.001)$. On comparison between the NRSPA and MA group, (A) shows significant differences in step length $(\mathrm{P}<0.05)$; (B) shows significant differences in step height $(\mathrm{P}<0.001)$; $(\mathrm{C})$ shows no significant difference in swing duration $(\mathrm{P}>0.05)$; and $(\mathrm{D})$ shows significant difference in step number $(\mathrm{P}<0.01)$. BWSTT, body-weight-supported treadmill training; NRSPA, normal rat stepping pattern assistance.

of steps was less than that of the intact rats, due to pain and weakness after laminectomy. The step cycle of the sham rats was consistent with that of the intact rats. The hindlimbs of all SCI rats could not perform stepping.

After 3 weeks of BWSTT, the rats in the NRSPA and MA groups gained greater locomotor recovery than the sedentary group in terms of longer horizontal movement (step length), higher vertical movement (step height), longer swing duration, and greater number of steps in the 30 -sec testing, although these results were significantly poorer than those recorded for the rats in the sham group. Compared to the MA group, the hindlimbs of the rats in the NRSPA group performed better stepping (Fig. 3). Factorial design ANOVA for stepping analysis showed that the source of the variation was different according to the different groups $(\mathrm{P}<0.001)$ but not according to the different hindlimbs $(\mathrm{P}>0.05)$. Therefore, in all rats, the right and left hindlimbs could perform consistently, and the differences in stepping quality improvement were due to the differences between the groups in step training schemes and assistive modes.

Step length (horizontal movement). Intact horizontal movements (left, $50.33 \pm 6.59 \mathrm{~mm}$; right, $51.3 \pm 5.37 \mathrm{~mm}$ ) were generated by the hindlimbs of the sham rats (Figs. 3A and 4A), which are consistent with those of the intact rats. The hindlimbs of sedentary rats performed occasional step-like movements (left, $5.87 \pm 2.04 \mathrm{~mm}$; right, $7.25 \pm 2.98 \mathrm{~mm}$ ) that could not be recognized as real stepping by RRMPS (Figs. 3B and 4A). The stepping patterns in the NRSPA and MA group were similar to those of the sham rats, although the amplitudes were smaller. This finding indicated that the rats benefited from BWSTT for improvement of the horizontal movement of the hindlimbs. The rats in the NRSPA group achieved a significantly longer step length (left, $25.08 \pm 3.06 \mathrm{~mm}$; right, $25.23 \pm 3.91 \mathrm{~mm}$; $\mathrm{P}<0.05$ ) (Figs. 3C and 4A) than those in the MA group (left, $18.54 \pm 2.84 \mathrm{~mm}$; right, $17.86 \pm 4.03 \mathrm{~mm}$ ) (Figs. 3D and 4A).

Step height (vertical movement). The hindlimbs of the sham rats performed normal vertical movement (left, $16.75 \pm 2.77 \mathrm{~mm}$; right, $17.78 \pm 2.42 \mathrm{~mm}$ ) (Figs. 3A and 4B) and the stepping was coordinated as the step height correlated with the step length. The sedentary rats performed minimal vertical movements (left, $0.81 \pm 0.23 \mathrm{~mm}$; right, $0.77 \pm 0.23 \mathrm{~mm}$ ), which were not recognized as step movements by the analysis software. The improvement in vertical movements among the NRSPA rats (left, $12.55 \pm 1.75 \mathrm{~mm}$; right, $12.52 \pm 1.41 \mathrm{~mm}$ ) (Fig. 3C) was significantly different $(\mathrm{P}<0.001)$ (Fig. 4B) than that recorded among the MA rats (left, $3.00 \pm 0.69 \mathrm{~mm}$; right, $3.07 \pm 1.07 \mathrm{~mm}$ ) (Figs. 3D and 4B). The vertical movements of the hindlimbs in the NRSPA group markedly improved and were repeated consistently, following the rhythm of movements of the forelimbs to some extent. However, the rats in 
A BBB scores variation during time transition

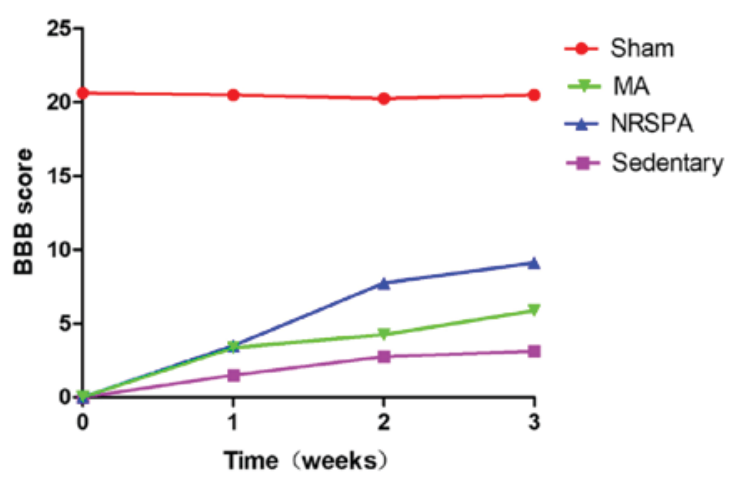

C

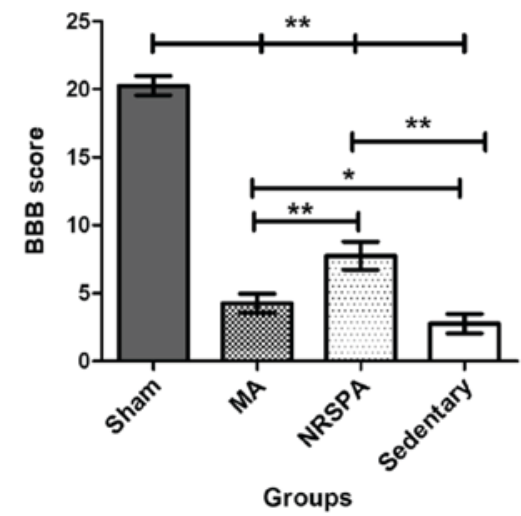

B BBB scores after 1 week BWSTT

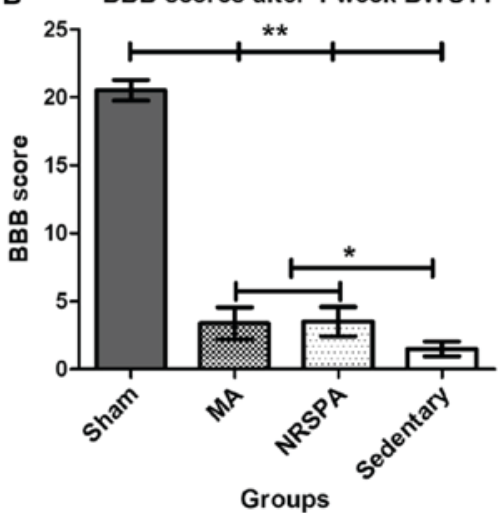

D BBB scores after 3 week BWSTT

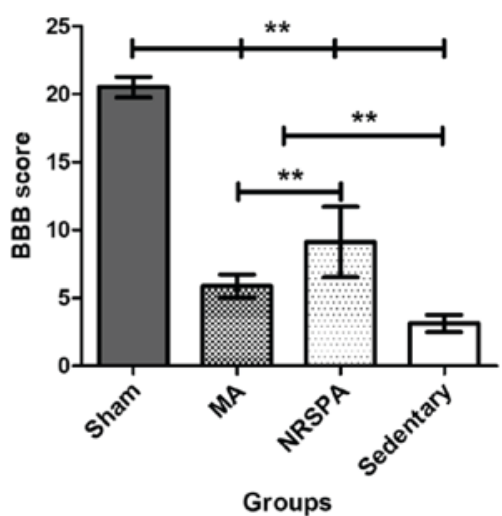

Figure 5. Comparison of BBB scores for all experimental groups following time transition. As shown in (A), BBB scores show a variation trend from the beginning to the end of the BWSTT. BBB scores of all the SCI groups showed a growth trend over time and the BBB score of the sham group remained stable. The BBB score of the NRSPA group increased faster than that of the MA group from the second week of BWSTT, although the BBB scores of the two groups were similar at the end of 1-week BWSTT. (B-D) show the comparison of BBB scores between the different groups at the end of 1,2, and 3 weeks of BWSTT, respectively. One-way ANOVA and LSD were used for statistical analysis and $\mathrm{P}<0.05$ was statistically significant. ${ }^{*} \mathrm{P}<0.01$ and ${ }^{* *} \mathrm{P}<0.001$. On comparison between the NRSPA and MA group, (B) shows no significant difference in BBB scores after the first week, while (C and D) show significant differences in BBB scores in the second and third week, respectively $(\mathrm{P}<0.001)$. BBB, Basso, Beattie, and Bresnahan; BWSTT, body-weight-supported treadmill training; SCI, spinal cord injury; NRSPA, normal rat stepping pattern assistance.

the MA group did not perform obvious vertical movements, which were disproportionate to the amplitude of horizontal movements (Fig. 3D).

Swing duration. Among the sedentary rats, no steps were recognized by the analysis software; therefore, the comparison of the swing duration only included the sham group, NRSPA group, and MA group. After 3 weeks of BWSTT, the increase in the swing duration in the NRSPA group (left, $0.35 \pm 0.04 \mathrm{sec}$; right, $0.35 \pm 0.03 \mathrm{sec}$ ) was greater than that in the MA group (left, $0.30 \pm 0.06 \mathrm{sec}$; right, $0.31 \pm 0.05 \mathrm{sec}$ ), although the difference was not statistically significant $(\mathrm{P}>0.05)$. In both groups, swing duration was significantly shorter $(\mathrm{P}<0.01)$ than that in the sham rats (left, $0.42 \pm 0.03 \mathrm{sec}$; right, $0.43 \pm 0.04 \mathrm{sec}$ ) (Fig. $4 \mathrm{C}$ ).

Step number. The hindlimbs of the sedentary rats generated some step-like movements but did not meet the criteria for complete steps. Following 3 weeks of BWSTT, the average number of complete steps in the 30-sec testing performed by the NRSPA group (left, 10.13 \pm 1.13 ; right, 10.75 \pm 1.04 ) and the MA group (left, 6.88 \pm 1.36 ; right, $6.75 \pm 1.28$ ) was significantly less than that of the sham rats (left, 15.63 \pm 1.69 ; right, $15.88 \pm 1.25)(\mathrm{P}<0.001)$. The number of complete steps in the 30-second testing performed by the NRSPA group was significantly greater than that of the MA group $(\mathrm{P}<0.01)$ (Fig. 4D).

Comparison of $B B B$ scores between the groups provided consistent evidence of step detection by RRMPS. All the rats presented normal locomotion before surgery. Three days after SCI, all the rats with spinal contusion had the lowest scores (0) and the rats in the sham group had the highest

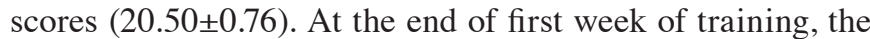
BBB scores increased slightly across all SCI groups, and the BWSTT groups (NRSPA group and MA group) had higher scores than the sedentary group (BBB score, 1.5 \pm 0.53 ) $(\mathrm{P}<0.01)$. No significant difference was found between the NRSPA group (BBB score, $3.5 \pm 1.07$ ) and the MA group (BBB score, 3.38 \pm 1.19$)(\mathrm{P}>0.05)$ (Fig. 5A and B). After 2 weeks of training, the BBB scores of the NRSPA group (BBB score, 6.35 \pm 0.74 ) increased significantly compared to the scores recorded during the first week, although no obvious improvement was observed in the MA group (BBB score, 4.63 \pm 0.52$)$; the difference between the two BWSTT groups was statistically significant $(\mathrm{P}<0.001)$ (Fig. 5A and $\mathrm{C}$ ). Assessment was repeated at the end of the third week of BWSTT and the BBB scores of the NRSPA group, the MA 


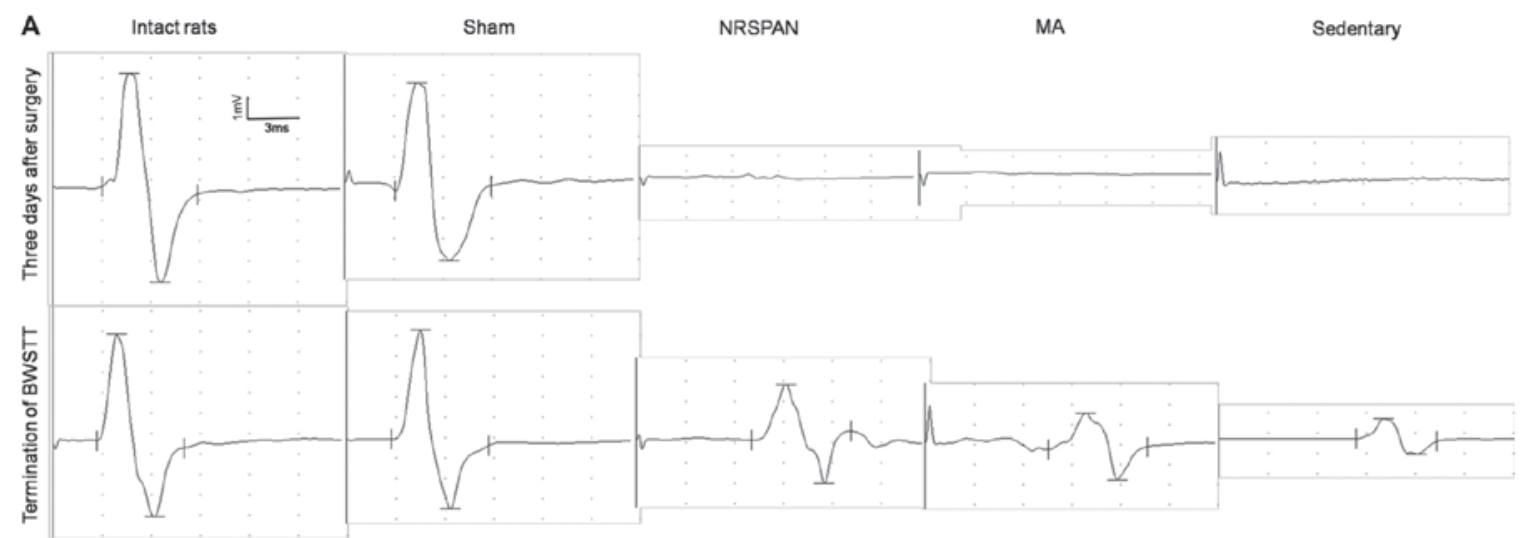

B Lantecy of tceMEPs after 3 week BWSTT

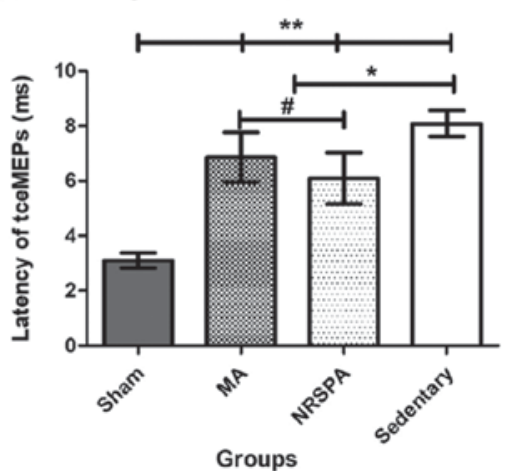

C Amplitude of tceMEPs after 3 week BWSTT

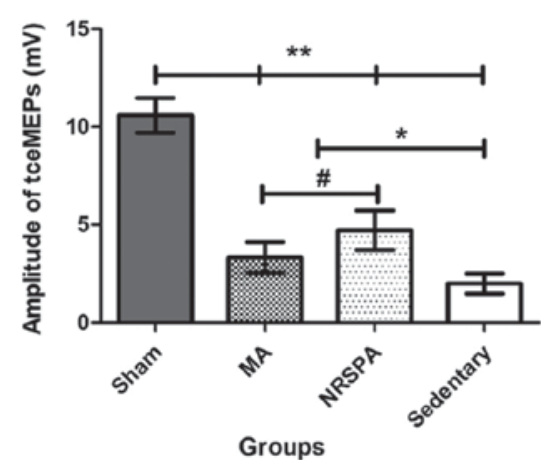

Figure 6. The representative tceMEPs waveforms at the beginning and the termination of BWSTT and the tceMEPs parameters analysis. (A) The variation trends of tceMEPs waveforms in all experimental groups by comparing the tceMEPs waveforms obtained at 3 days after surgery and the termination of the 3 weeks of BWSTT. The sham group had exactly the same waveform as the intact rats. The tceMEPs waveforms could not be detected in the three SCI groups (NRSPA, MA and sedentary) 3 days after surgery. Following a 3-week BWSTT, the latencies and amplitudes of tceMEPs waveform in NRSPA group gained the greatest increase in contrast to those of the MA and sedentary groups. (B and C) The results of statistical analysis for latency and amplitude of tceMEPs, respectively. One-way ANOVA and LSD were used for statistical analysis and $\mathrm{P}<0.05$ was statistically significant. ${ }^{*} \mathrm{P}<0.05,{ }^{*} \mathrm{P}<0.01,{ }^{* *} \mathrm{P}<0.001$. (B) represents significant differences in the latency of tceMEPs between the NRSPA group and MA group (P<0.05). (C) The significant differences in the amplitude of tceMEPs between the two BWSTT groups (NRSPA and MA group) $(\mathrm{P}<0.05)$. tceMEPs, transcranial electrical motor-evoked potentials; BWSTT, body-weight-supported treadmill training; SCI, spinal cord injury; NRSPA, normal rat stepping pattern assistance; MA, manual assistance.

group, and the sedentary group were significantly lower than those of the sham group which exhibited normal continuous plantar stepping $(\mathrm{P}<0.001)$. The sedentary rats did not regain further functional activity and their hindlimbs exhibited minimal joint movement (BBB score, $3.13 \pm 0.45)$. The $\mathrm{BBB}$ scores of the MA group (BBB score, 5.88 \pm 0.83 ) were significantly lower than those of the NRSPA group (BBB score, 9.13 \pm 2.59$)(\mathrm{P}<0.001)$ (Fig. 5A and D). After 3 weeks of training, the rats in the NRSPA group showed occasional plantar stepping without weight support and coordinated forelimb gait. The rats in the MA group showed coordinated and extensive movements of the hip-joint and knee-joint and slight movement of the ankle-joint of the hindlimb, but no plantar stepping and weight support.

Electrophysiological assessments demonstrated uneven improvement between the groups. The waveforms of tceMEPs were not detectable in all SCI groups (NRSPA, MA, sedentary) 3 days after surgery (Fig. 6A). No significant changes in latency and amplitude were found in the sham group compared to the baseline of intact rats throughout the whole procedure. After 3 weeks of BWSTT, the waveforms of tceMEPs were measured in the different SCI groups. The latency and amplitude were restored slightly in the sedentary rats, which were significantly different to the sham rats $(\mathrm{P}<0.001)$ and the two BWSTT groups $(\mathrm{P}<0.01)$ (Fig. 6B and $\mathrm{C})$. The two BWSTT groups with different assistive modes presented shorter latency and taller amplitude compared to the sedentary group (Fig. 6A), which indicated that the electrical signal conduction function of the spinal cord was improved by BWSTT. Furthermore, the rats in the NRSPA group exhibited better conduction parameters than the rats in MA group $(\mathrm{P}<0.05)$ (Fig. $6 \mathrm{~B}$ and $\mathrm{C}$ ).

NF200 protein expression in spinal cord lesion area. After the 3 -week experimental period, the mean density (MD) and positive area fraction (AF) of $\mathrm{NF}_{200^{+}}$expression in the dorsal horn of the spinal cord lesion center were analyzed and compared between groups. The AF and MD values of the sham group were significantly higher than those in all the other SCI groups $(\mathrm{P}<0.001)$ (Figs. 7 and 8$)$. The AF of $\mathrm{NF}_{200}{ }^{+}$expression in the two BWSTT groups (NRSPA and MA) increased significantly compared to the sedentary group $(\mathrm{P}<0.05)$, although expression remained significantly below the normal level $(\mathrm{P}<0.001)$ (Fig. 8B). There was no significant difference in the AF values between the NRSPA group and MA group ( $\mathrm{P}>0.05)$ (Fig. 8B). A significant difference in MD values was found between the sedentary group and the two BWSTT groups (MA group, $\mathrm{P}<0.05$; NRSPA group, $\mathrm{P}<0.01$ ). 
A

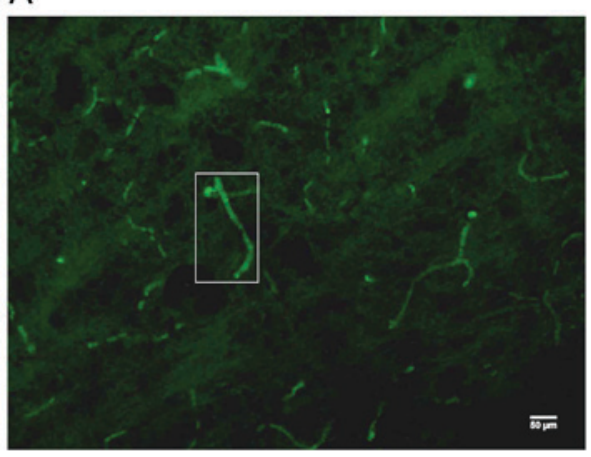

C

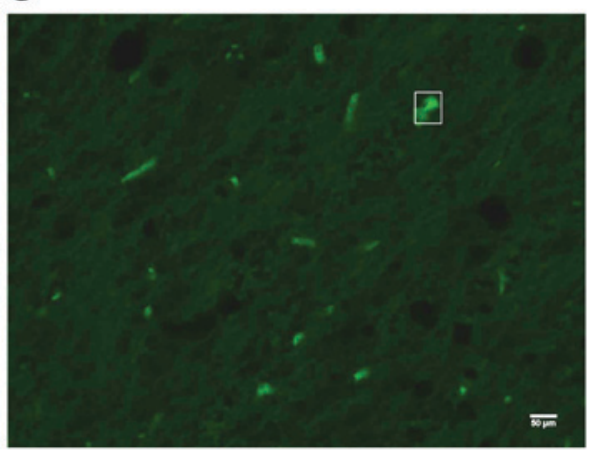

B

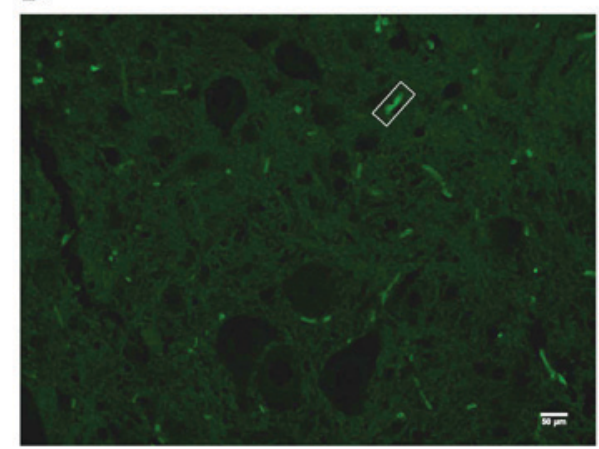

D

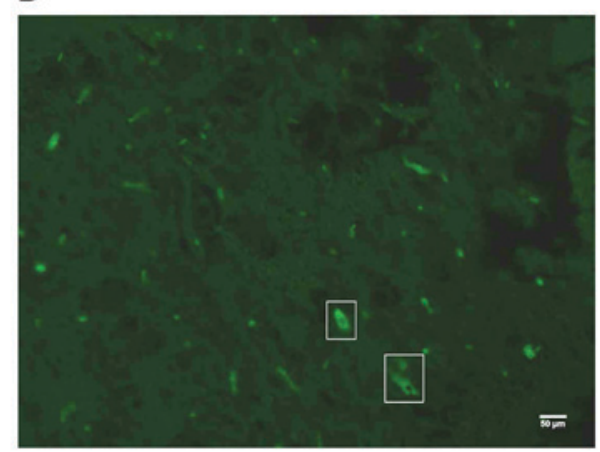

Figure 7. The representative images of $\mathrm{NF}_{200}{ }^{+}$expression under a 20X objective lens in the dorsal horn of the spinal cord lesion epicenter after 3 weeks of BWSTT. (A-D) NF200+ expression for the sham, sedentary, NRSPA, and MA groups, respectively, and the green fluorescence in the white boxes is typical NF200 $0^{+}$expression. (A) The extensive axonal connection is visible in the sham group, while in (B) the NF200 ${ }^{+}$expression is scattered, short, and thin in the sedentary group. Scattered, short and dotted NF $200^{+}$expression with limited strong positive expression can been seen in the NRSPA (C) and MA groups (D); the differences between the two groups are not obvious, as shown in the image. NF200, neurofilament 200; BWSTT, body-weight-supported treadmill training; NRSPA, normal rat stepping pattern assistance; MA, manual assistance.

A Mean density (MD) of NF200 ${ }^{+}$expression in dorsal columns of spinal cord lesion centre

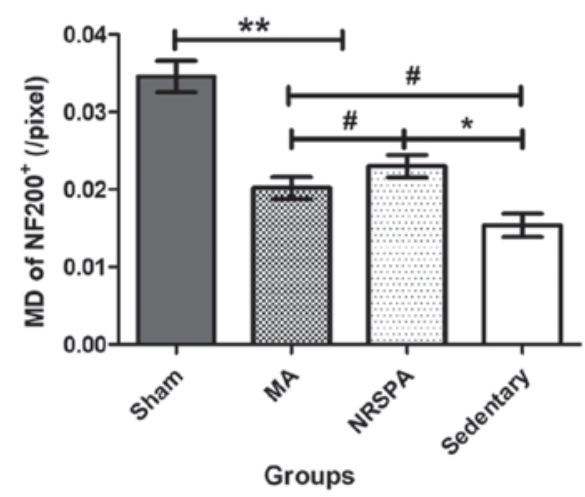

B Area fraction (AF) of $\mathrm{NF}_{200^{+}}$expression in dorsal columns of spinal cord lesion centre

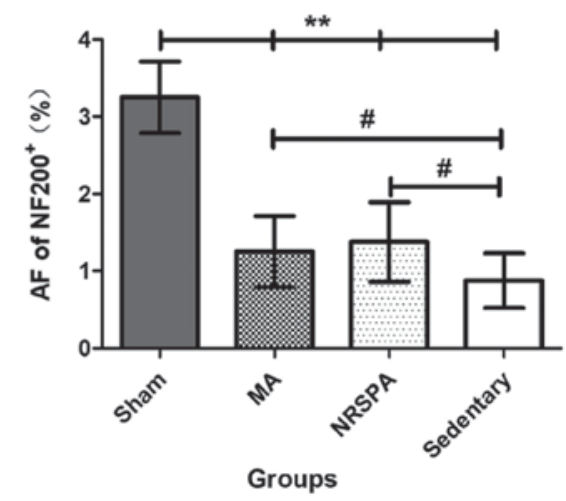

Figure 8. Comparison between the quantitative analysis based on MD and AF of NF200 expression. The charts represent the outcomes of the quantitative analysis for $\mathrm{AF}$ and $\mathrm{MD}$ for $\mathrm{NF}_{200}{ }^{+}$expression and comparison between the groups. One-way ANOVA and LSD were used for statistical analysis and $\mathrm{P}<0.05$ was statistically significant. ${ }^{~} \mathrm{P}<0.05,{ }^{*} \mathrm{P}<0.01,{ }^{* *} \mathrm{P}<0.001$. (A) The significant differences in MD between the NRSPA and MA group ( $\left.\mathrm{P}<0.05\right)$. (B) No significant difference in AF between the NRSPA and MA group. MD, mean density; AF, area fraction; NRSPA, normal rat stepping pattern assistance; MA, manual assistance.

The MD values in the MA group were significantly lower than those in the NRSPA group $(\mathrm{P}<0.05)$ (Fig. 8A).

\section{Discussion}

In this study, we compared two assistive training patterns for the hindlimbs during BWSTT to assess the improvement of the locomotor function, nerve conduction function, and nerve regeneration in the spinal cord based on a rat model of acute SCI. We demonstrated that the rats in the NRSPA group achieved better recovery in terms of stepping function, neural conduction of the spinal cord, and axon regeneration than the rats in the MA group. These results showed that NRSPA for the hindlimbs was more effective than MA during BWSTT for the improvement of axon regeneration and neural conduction in the early phase of acute SCI. 
It is recognized that some degree of locomotor function of the hindlimbs can recover spontaneously in rats with incomplete SCI. This is consistent with the findings of this study that the BBB scores and kinematic parameters of the hindlimbs in the sedentary group increased gradually without any exercise training. However, recovery was limited for the outcomes of step analysis as the hindlimb movements of sedentary rats did not meet the stepping criteria for height and length of hindlimb movement. The hindlimbs of the rats in the two BWSTT groups achieved considerable improvement in locomotor function after 3 weeks of step training, indicating that BWSTT with assistance to the paralyzed limbs is an effective method for SCI rehabilitation in the early phase of acute SCI.

With advances in technology, rehabilitation interventions based on the use of robotics have been widely implemented, demonstrating trends for strong growth over the past two decades (35). Multiple studies have proved the effectiveness of robotic assistance to improve motor function $(23,36,37)$ and it is widely recognized that robotics are efficient substitutes of physiotherapists (36). It is not clear from the available research on humans which are the most effective (38) although animal studies are scarce. Theoretically, robotic assistance under computer control, should be more stable while MA is more variable. Natural walking is characterized by variability and inconsistency in step length, step height, step speed, and center of mass dur to variable environmental features; therefore, the training is varied and fits the features of natural walking. Thus, it is increasingly recognized that movement variability is an essential requirement for skilled, adaptable movements $(11,39)$. It is increasingly being recognized that the practice occurring to achieve these skilled movements must allow for variability (40). Therefore, unlike MA, the lack of variability of robotic assistance has been a criticism of robotic assistance. However, from another perspective, the spinal cord is able to sustain several forms of learning and memory, including limb-position training and several forms of adaptive motor plasticity, which can generate profound effects on locomotor behavior. Therefore, the lack of variability and the precise repetition of training cycles may be advantages of robotic assistance because the precise movements with high number of repetitions are recognized as beneficial to learning (35). In this study, we used the BWSTT platform to compare the effects of different assistance training patterns for BWSTT. One of these is assistance by robotic arms following a trajectory of normal rat hindlimb stepping (NRSPA) which has been shown to be consistent (Table I and Fig. 1). The other is assistance by the hands of an experienced trainer (MA) who can assist the hindlimbs of the SCI rats as evenly as possible. From the outcomes of the BBB score over 3 weeks of training, the rats in the NRSPA group achieved greater improvements than those in the MA group at the end of the second and third week of training. Although no differences were found between the BBB scores of the NRSPA group and those of the MA group after the first week of training, the average BBB score in the NRSPA group was higher than that of in the MA group. The results of step detection and analysis also supported the above outcomes, the hindlimbs of rats in the NRSPA group gained longer horizontal movement (step length), higher vertical movement (step height), longer swing duration and more step numbers (Fig. 4). Based on the above locomotor function evaluation results, the NRSPA achieved consistent and stable characteristics more effectively than the MA. However, locomotor function recovery in the early stages of SCI is the most effective time for step learning rather than accommodating skilled movements.

Generally, locomotion in mammals, including humans, is based on the activity of neuronal circuits within the spinal cord. It has been proved that incomplete lesions to the spinal cord are accompanied by axonal sprouting in the vicinity of the lesion within weeks of SCI (41). Post-SCI axonal sprouting can form new intraspinal neuronal circuits that allow descending pathways to bypass the site of the lesion. Recent studies have shown that exercise training can enhance axon sprouting following SCI (42) and axon regrowth remains the major prerequisite for plasticity, regeneration, circuit formation, and eventually functional recovery (43). Recent studies have suggested a plastic behavior of the spinal neuronal circuits (44-46) and alteration of the step characteristics (47) were observed following SCI after locomotor training, that is, the relearning of the spinal cord. However, this relearning process of the spinal cord is affected by the pattern of stimuli during training, and noncontingent stimuli can prevent future attempts at learning through a central sensitization mechanism (48). Therefore, theoretically, the activity-dependent plasticity within the spinal cord should be carefully modified to promote adaptive spinal training. Stimulation delivered in a limb position-dependent manner or at a fixed interval are able to induce adaptive plasticity that promotes spinal cord learning (30). Based on the plasticity mechanism of spinal neuronal circuits, we hypothesized that NRSPA may be a more effective stimulus than MA because NRSPA was congruent with the normal stepping pattern. To prove our hypothesis, we used the tceMEPs assessment which was an objective assessment of electrical conduction through the associated neural pathways (49-51) and immunohistochemistry analysis for neurofilament protein NF200 (NF200) expression, which was the main component of the neuronal and axonal cytoskeleton. By tceMEPs assessment, the waveforms of tceMEPs have not be detected 3 days after surgery in all the SCI rats for the acute injury at the T10 level. After 3 weeks of BWSTT, the tceMEPs waveforms in the NRSPA group demonstrated greater improvement compared to the MA group for shorter latencies and greater amplitudes (Fig. 6), indicating greater neural conduction in the spinal cord than the later. The quantitative analysis for $\mathrm{NF}_{200^{+}}$expression by immunohistochemistry in the dorsal horn of the spinal cord lesion epicenter was performed to validate the tceMEPs set-up. Our results indicated that the positive area fraction (AF) and mean density (MD) of the NF200 ${ }^{+}$expression in the two BWSTT groups (NRSPA and MA) increased significantly compared to those of the sedentary group $(\mathrm{P}<0.05)$ (Figs. 7 and 8$)$. Importantly, the MD values of the NF200-labeled axons in the MA group were significantly lower than those in the NRSP group, although there was no statistically significant difference in AF values between the two BWSTT groups. Therefore, on the basis of the results of the tceMEPs assessment and NF200 immunoreactivity, we conclude that the rats undergoing BWSTT and receiving NRSPA can achieve greater nerve regeneration and neural conduction in the spinal cord than those undergoing BWSTT and receiving MA.

In conclusion, NRSPA for paralytic hindlimbs was more effective than MA in promoting the stepping learning process 
during BWSTT in the early phase of acute SCI. This finding is demonstrated by improved locomotor function evaluation, neural conduction, and nerve regeneration of the spinal cord lesion area. These results indicate that the establishment of an accurate assistive pattern of training to correct the subtle mistakes during stepping learning from the beginning is critical. However, stepping is a complex movement involving controlling the central nervous system and the functioning of a group of skeletal muscles, joints, and limb coordination, and the different training duration and training intensity may influence the results to some extent. All the above parts were not evaluated in this study. Therefore, further studies are required to validate the different effects of NRSPA and MA from a more comprehensive perspective.

We examined the effects of NRSPA for robotic-assisted treadmill training on locomotor recovery in rats with SCI. Spinally contused rats receiving NRSPA during BWSTT performed better hindlimb stepping and had greater tceMEPs recovery and $\mathrm{NF}_{200^{+}}$expression in the spinal cord lesion area than rats receiving MA. This new assistive pattern of training for BWSTT is potentially a better platform support for animal experiments.

\section{Acknowledgements}

We are thankful for the support of all staff from the Experimental Center of the School of Nursing, Dalian University in China. This study was funded by the Education Department of Liaoning Province (grant no. L2014489) and the Science and Technology Department of Liaoning Province (grant no. 201602022).

\section{References}

1. Macias M, Dwornik A, Ziemlinska E, Fehr S, Schachner M, Czarkowska-Bauch J and Skup M: Locomotor exercise alters expression of pro-brain-derived neurotrophic factor, brain-derived neurotrophic factor and its receptor TrkB in the spinal cord of adult rats. Eur J Neurosci 25: 2425-2444, 2007.

2. Macias M, Nowicka D, Czupryn A, Sulejczak D, Skup M, Skangiel-Kramska J and Czarkowska-Bauch J: Exercise-induced motor improvement after complete spinal cord transection and its relation to expression of brain-derived neurotrophic factor and presynaptic markers. BMC Neurosci 10: 144, 2009.

3. Rangasamy SB: Locomotor recovery after spinal cord hemisection/contusion injures in bonnet monkeys: Footprint testing-a minireview. Synapse 67: 427-453, 2013.

4. Smith RR, Brown EH, Shum-Siu A, Whelan A, Burke DA, Benton RL and Magnuson DS: Swim training initiated acutely after spinal cord injury is ineffective and induces extravasation in and around the epicenter. J Neurotrauma 26: 1017-1027, 2009.

5. Onifer SM, Zhang O, Whitnel-Smith LK, Raza K, O'Dell CR, Lyttle TS, Rabchevsky AG, Kitzman PH and Burke DA: Horizontal ladder task-specific re-training in adult rats with contusive thoracic spinal cord injury. Restor Neurol Neurosci 29: 275-286, 2011.

6. Côté MP, Azzam GA, Lemay MA, Zhukareva V and Houlé JD: Activity-dependent increase in neurotrophic factors is associated with an enhanced modulation of spinal reflexes after spinal cord injury. J Neurotrauma 28: 299-309, 2011.

7. de Leon RD, See PA and Chow CH: Differential effects of low versus high amounts of weight supported treadmill training in spinally transected rats. J Neurotrauma 28: 1021-1033, 2011.

8. Watanabe S, Oya Y, Iwata J and Someya F: Influences of changes in the level of support and walking speed on the $\mathrm{H}$ Reflex of the soleus muscle and circulatory dynamics on body weight-supported treadmill training: investigation in healthy adults. J Phys Ther Sci 26: 1345-1350, 2014.
9. Lee SW, Kim YS, Jun TW, Seo JH, Kim K, Shin MS and Kim CJ: The impact of duration of one bout treadmill exercise on cell proliferation and central fatigue in rats. J Exerc Rehabil 9: 463-469, 2013.

10. Kim YP, Kim HB, Jang MH, Lim BV, Kim YJ, Kim H, Kim SS, Kim EH and Kim CJ: Magnitude- and time-dependence of the effect of treadmill exercise on cell proliferation in the dentate gyrus of rats. Int J Sports Med 24: 114-117, 2003.

11. Shah PK, Gerasimenko Y, Shyu A, Lavrov I, Zhong H, Roy RR and Edgerton VR: Variability in step training enhances locomotor recovery after a spinal cord injury. Eur J Neurosci 36: 2054-2062, 2012.

12. Navarrete-Opazo A, Alcayaga JJ, Sepúlveda O and Varas G: Intermittent hypoxia and locomotor training enhances dynamic but not standing balance in patients with incomplete spinal cord injury. Arch Phys Med Rehabil 98: 415-424, 2017.

13. Kamgar P, Agarwal A, Chao T, Askari S, Tan M, Honor R and Won DS: Step trajectory analysis of spinal cord injured rats trained with neuromuscular electrical stimulation coordinated with robotic treadmill training. Conf Proc IEEE Eng Med Biol Soc 2012: 1864-1867, 2012.

14. Scivoletto G, Tamburella F, Laurenza L, Torre $M$ and Molinari M: Who is going to walk? A review of the factors influencing walking recovery after spinal cord injury. Front Hum Neurosci 8: 141, 2014.

15. Martinez M, Delivet-Mongrain H and Rossignol S: Treadmill training promotes spinal changes leading to locomotor recovery after partial spinal cord injury in cats. J Neurophysiol 109: 2909-2922, 2013

16. Battistuzzo CR, Rank MM, Flynn JR, Morgan DL, Callister R, Callister RJ and Galea MP: Effects of treadmill training on hindlimb muscles of spinal cord-injured mice. Muscle Nerve 55: 232-242, 2017

17. Heng $\mathrm{C}$ and de Leon RD: Treadmill training enhances the recovery of normal stepping patterns in spinal cord contused rats. Exp Neurol 216: 139-147, 2009.

18. Yang JF, Musselman KE, Livingstone D, Brunton K, Hendricks G, Hill D and Gorassini M: Repetitive mass practice or focused precise practice for retraining walking after incomplete spinal cord injury? A pilot randomized clinical trial. Neurorehabil Neural Repair 28: 314-324, 2014.

19. Battistuzzo CR, Callister RJ, Callister R and Galea MP: A systematic review of exercise training to promote locomotor recovery in animal models of spinal cord injury. J Neurotrauma 29: 1600-1613, 2012.

20. Norrie BA, Nevett-Duchcherer JM and Gorassini MA: Reduced functional recovery by delaying motor training after spinal cord injury. J Neurophysiol 94: 255-264, 2005.

21. Panisset MG, Galea MP and El-Ansary D: Does early exercise attenuate muscle atrophy or bone loss after spinal cord injury? Spinal Cord 54: 84-92, 2016.

22. Hornby TG, Zemon DH and Campbell D: Robotic-assisted, body-weight-supported treadmill training in individuals following motor incomplete spinal cord injury. Phys Ther 85: 52-66, 2005 .

23. Gorman PH, Scott W, York H, Theyagaraj M, Price-Miller N, McQuaid J, Eyvazzadeh M, Ivey FM and Macko RF: Robotically assisted treadmill exercise training for improving peak fitness in chronic motor incomplete spinal cord injury: A randomized controlled trial. J Spinal Cord Med 39: 32-44, 2016.

24. Lee C, Won D, Cantoria MJ, Hamlin M and de Leon RD: Robotic assistance that encourages the generation of stepping rather than fully assisting movements is best for learning to step in spinally contused rats. J Neurophysiol 105: 2764-2771, 2011.

25. Aoyagi D, Ichinose WE, Harkema SJ, Reinkensmeyer DJ and Bobrow JE: A robot and control algorithm that can synchronously assist in naturalistic motion during body-weight-supported gait training following neurologic injury. IEEE Trans Neural Syst Rehabil Eng 15: 387-400, 2007.

26. Emken JL, Harkema SJ, Beres-Jones JA, Ferreira CK and Reinkensmeyer DJ: Feasibility of manual teach-and-replay and continuous impedance shaping for robotic locomotor training following spinal cord injury. IEEE Trans Biomed Eng 55: 322-334, 2008.

27. Vallery H, van Asseldonk EH, Buss M and van der Kooij H: Reference trajectory generation for rehabilitation robots: Complementary limb motion estimation. IEEE Trans Neural Syst Rehabil Eng 17: 23-30, 2009. 
28. Hornby TG, Campbell DD, Kahn JH, Demott T, Moore JL and Roth HR: Enhanced gait-related improvements after therapist-versus robotic-assisted locomotor training in subjects with chronic stroke: A randomized controlled study. Stroke 39: 1786-1792, 2008.

29. Hussain S, Xie SQ and Liu G: Robot assisted treadmill training: mechanisms and training strategies. Med Eng Phys 33: 527-533, 2011.

30. Shah PK, Garcia-Alias G, Choe J, Gad P, Gerasimenko Y, Tillakaratne N, Zhong H, Roy RR and Edgerton VR: Use of quadrupedal step training to re-engage spinal interneuronal networks and improve locomotor function after spinal cord injury. Brain 136: 3362-3377, 2013.

31. Basso DM, Beattie MS and Bresnahan JC: A sensitive and reliable locomotor rating scale for open field testing in rats. J Neurotrauma 12: 1-21, 1995.

32. Wang D and Zhang J: Electrophysiological functional recovery in a rat model of spinal cord hemisection injury following bone marrow-derived mesenchymal stem cell transplantation under hypothermia. Neural Regen Res 7: 749-755, 2012.

33. Li WT,Zhang XY, Xue H, Ni CP, Wang EG and An LB: Comparison of three different time points of starting treadmill training in spinal cord injured rats. Dev Neurorehabil 16: 382-390, 2013.

34. Chen G, Zhang Z, Wang S and Lv D: Combined treatment with FK506 and nerve growth factor for spinal cord injury in rats. Exp Ther Med 6: 868-872, 2013.

35. Esquenazi A and Packel A: Robotic-assisted gait training and restoration. Am J Phys Med Rehabil 91 (11 Suppl 3): S217-S231, 2012.

36. Shin JC, Kim JY, Park HK and Kim NY: Effect of robotic-assisted gait training in patients with incomplete spinal cord injury. Ann Rehabil Med 38: 719-725, 2014.

37. Schwartz I and Meiner Z: Robotic-assisted gait training in neurological patients: Who may benefit. Ann Biomed Eng 43: $1260-1269,2015$

38. Srivastava S, Kao PC, Reisman DS, Scholz JP, Agrawal SK and Higginson JS: Robotic assist-as-needed as an alternative to therapist-assisted gait rehabilitation. Int J Phys Med Rehabil 4 $370,2016$.

39. Harbourne RT and Stergiou N: Movement variability and the use of nonlinear tools: Principles to guide physical therapist practice. Phys Ther 89: 267-282, 2009.

40. Fetters L: Perspective on variability in the development of human action. Phys Ther 90: 1860-1867, 2010.

41. Bareyre FM, Kerschensteiner M, Raineteau O, Mettenleiter TC, Weinmann O and Schwab ME: The injured spinal cord spontaneously forms a new intraspinal circuit in adult rats. Nat Neurosci 7 : $269-277,2004$
42. Houle JD and Côté MP: Axon regeneration and exercise-dependent plasticity after spinal cord injury. Ann N Y Acad Sci 1279 154-163, 2013.

43. Filous AR and Schwab JM: Determinants of axon growth, plasticity and regeneration in the context of spinal cord injury. Am J Pathol 188: 53-62, 2018.

44. Gossard JP, Delivet-Mongrain H, Martinez M, Kundu A, Escalona M and Rossignol S: Plastic changes in lumbar locomotor networks after a partial spinal cord injury in cats. J Neurosci 35: 9446-9455, 2015.

45. Martinez M, Delivet-Mongrain H, Leblond $\mathrm{H}$ and Rossignol S: Incomplete spinal cord injury promotes durable functional changes within the spinal locomotor circuitry. J Neurophysiol 108: 124-134, 2012.

46. Adkins DL, Boychuk J, Remple MS and Kleim JA: Motor training induces experience-specific patterns of plasticity across motor cortex and spinal cord. J Appl Physiol (1985) 101: 1776-1782, 2006.

47. Rossignol S and Frigon A: Recovery of locomotion after spinal cord injury: Some facts and mechanisms. Annu Rev Neurosci 34: 413-440, 2011

48. Liu GT, Ferguson AR, Crown ED, Bopp AC, Miranda RC and Grau JW: Instrumental learning within the rat spinal cord: Localization of the essential neural circuit. Behav Neurosci 119: 538-547, 2005.

49. Morris SH, Howard JJ, Rasmusson DD and El-Hawary R: Validity of transcranial motor evoked potentials as early indicators of neural compromise in rat model of spinal cord compression. Spine (Phila Pa 1976) 40: E492-E497, 2015.

50. Alam M, Garcia-Alias G, Shah PK, Gerasimenko Y, Zhong H, Roy RR and Edgerton VR: Evaluation of optimal electrode configurations for epidural spinal cord stimulation in cervical spinal cord injured rats. J Neurosci Methods 247: 50-57, 2015.

51. Petersen JA, Spiess M, Curt A, Dietz V and Schubert M; EM-SCI Study Group: Spinal cord injury: One-year evolution of motor-evoked potentials and recovery of leg motor function in 255 patients. Neurorehabil Neural Repair 26: 939-948, 2012.

This work is licensed under a Creative Commons Attribution-NonCommercial-NoDerivatives 4.0 International (CC BY-NC-ND 4.0) License. 